\title{
Bending behavior of Cu-plated Pd-Ni alloys ribbon driven by
}

\section{hydrogenation}

\author{
Masayuki Mizumoto $^{\mathrm{a}, *}$, Takeshi Ohgai ${ }^{\mathrm{a}}$ and Akio Kagawa ${ }^{\mathrm{a}}$ \\ ${ }^{a}$ Department of Materials Science and Engineering, Nagasaki University, 1-14 Bunkyo- \\ machi, Nagasaki 852-8131, JAPAN
}

\begin{abstract}
In recent years, human-type robots attract much attention in the field of medical welfare and industrial applications under severe environmental conditions, such as in space. For the realization of such robots, the development of an actuator having superior functions as human fingers is required. It is known that hydrogen storage alloys exhibit a significant volume change of $10-30 \%$ on hydrogenation, which is several tens times greater than that caused by thermal expansion. Since the driving force of an actuator driven by hydrogenation is based on phase transformation, the actuator can be expected to show high power. In the present work, a bending module having a sheet form of palladium-base hydrogen storage alloy foils bond with a copper foil was prepared and its bending behaviors were investigated.
\end{abstract}

* Corresponding author Tel: +81-95-819-2641, fax: +81-95-819-2641.

E-mail address: mizumoto@nagasaki-u.ac.jp 
Keywords: hydrogen absorbing materials; palladium; actuator

\section{Introduction}

It is well known that hydrogen storage alloys (HSA) can reversibly absorb and desorb a large amount of hydrogen, about 1000 times greater than their own volume and exhibit a significant volume change of $10-30 \%$ on hydrogenation [1]. Palladium-base HSAs used in this study have some advantages, i.e. high pulverization resistance on hydrogen absorption - desorption cycles, easy activation, enough ductility to form into foils, in comparison with intermetallic compound alloys such as $\mathrm{LaNi}_{5}$ alloy [2, 3]. Therefore, the huge expansion on hydrogenation can be used as a driving force for actuators shown in Fig. 1. In comparison with thermal expansion of a bimetal (the thermal expansion coefficient is of the order of $10^{-5} / \mathrm{K}$ ), the expansion on hydrogen absorption is several tens times greater than that caused by thermal expansion. In addition, hydrogen absorption pressure (plateau pressure in the pressure - composition isotherm) can be set nearly 1 atm by choosing alloy compositions and let hydrogen desorb by reducing ambient hydrogen pressure at room temperature [4, 5]. In the present work, bending behavior of the actuator having a sheet form of HSAs bond with a copper foil was investigated. 


\section{Experimental Procedure}

Pd-11at\%Ni alloys were prepared by arc-melting pure materials (palladium> 99.9 wt. $\%$ and nickel> 99.9 wt.\%) under argon gas atmosphere. The alloy samples were annealed at $1073 \mathrm{~K}$ for 15 hours under vacuum for homogenization. After repeated rolling and annealing for stress relieving, rectangular sheet samples of $3 \mathrm{~mm} \times 50 \mathrm{~mm} \times$ $40 \mu \mathrm{m}$ was cut off from the rolled sheets. Copper-electroplating was performed on one side of the samples to convert the volume change of a HSA to bending motion. Plating thickness was $30-40 \mu \mathrm{m}$. The plated sample was maintained between stainless steel plates and was subjected to diffusion bonding heat -treatment at $1073 \mathrm{~K}$ for 2 hours under vacuum. Then, the sample was set in a shape change measurement apparatus shown in Fig. 2 and was heat-treated at $673 \mathrm{~K}$ for 3 hours in vacuo followed by an exposure to hydrogen gas of $3 \mathrm{~atm}$ for activation. The bending behavior of the sample was observed with a CCD camera.

\section{Results and Discussion}

Figure 3 shows pressure-composition isotherms of $\mathrm{Pd}-11$ at $\% \mathrm{Ni}$ alloy. By alloying with nickel, the plateau pressures of palladium base alloys for hydrogen absorption and desorption can be controlled and $\mathrm{Pd}-11 \mathrm{at} \% \mathrm{Ni}$ alloy has a desirable hydrogenation characteristic, i.e. flat plateau pressures nearly 1 atm and a small hysteresis on hydrogen absorption and desorption. Figure 4 shows a bending behavior 
of Pd-11at $\% \mathrm{Ni}$ alloy on hydrogen absorption. The left side of the sample is $\mathrm{Pd}-11 \mathrm{at} \% \mathrm{Ni}$ alloy and the right side is the $\mathrm{Cu}$-plating. The sample bends toward the plating side and the top end of the sample was moved about $13 \mathrm{~mm}$ in a horizontal direction in $45 \mathrm{~min}$. In addition, the Pd-11at $\% \mathrm{Ni}$ alloy showed a recover of displacement in vacuo after a hydrogen-absorbed sample was held in air for $24 \mathrm{~h}$. Figure 5 shows the displacement of the top end of the Pd-11at\%Ni alloy sample with time during hydrogen absorption desorption cycles. On each cycle, the response time required for the displacement of 7.5 $\mathrm{mm}$ was measured. In the first cycle, the bending displacement of $7.5 \mathrm{~mm}$ required about 6 minutes after hydrogen introduction. The displacement completely recovered in 45 minutes after evacuation up to $10^{-5}$ Torr. In comparison with the absorption process, the bending speed was greatly reduced on the desorption process. Figure 6 shows a response time of bending motion with the number of hydrogen absorption-desorption cycles for Pd-11at\%Ni alloy sample. Decrease in bending speed with number of cycles is observed in both absorption and desorption processes. This delay of response is considered to be caused by Up-hill diffusion [7, 8], that is, hydrogen diffuses toward a higher concentration portion of the sample. It is known in the earlier work that a reduction in thickness of a sample is effective to diminish the effects of Up-hill diffusion and thereby results in an increase of bending speed of the sample. Therefore, the sample thickness and the plating thickness were changed from $40 \mu \mathrm{m}$ to $20 \mu \mathrm{m}$. Figure 7 shows an appearance of Pd-11at\%Ni alloy sample with HSA thickness and 
plating thickness of $20 \mu \mathrm{m}$. The sample started to bend after hydrogen introduction, and about $21 \mathrm{~mm}$ displacement was observed in a horizontal direction after 60 seconds. The response time of the sample with HSA $20 \mu \mathrm{m}$ thick was improved significantly in comparison with that of the sample with HSA $40 \mu \mathrm{m}$ thick. Then, to investigate the effect of the strain induced by the bending motion of the sample, the strain of the sample after hydrogenation was estimated by the equation 1 .

$\varepsilon=t / 2 \rho$

where $\rho$ is the radius of curvature at the mid point and $t$ is a thickness of the sample. The $t$ value can be set to be equal to the distance between the interface and the exterior of the sample. The calculated $\varepsilon$ value for the sample with HSA $20 \mu \mathrm{m}$ thick and $40 \mu \mathrm{m}$ thick was $7.3 \times 10^{-4}$ and $7.9 \times 10^{-4}$, respectively. These calculated $\varepsilon$ values were small enough that the deformation of HSA and Cu-plating with bending motion of both samples would be within elastic area. Figure 8 shows a variation in displacement of the top end of the samples with time on hydrogen absorption. The sample with HAS thickness and Cu-plating thickness of $40 \mu \mathrm{m}$ shows a displacement of $20 \mathrm{~mm}$ at about $600 \mathrm{sec}$, while the sample with HSA thickness and Cu-plating thickness of $20 \mu \mathrm{m}$ shows the same displacement in $60 \mathrm{sec}$, showing 10 times faster bending speed due to a reduction of sample thickness. The following well-known equation gives a diffusion time, $t$, as a function of diffusion coefficient, $D$ and diffusion distance, $L$.

$t \propto L^{2} / D$ 
Since a diffusion coefficient is to be equal at room temperature, the diffusion time for the sample with HAS thickness of $40 \mu \mathrm{m}$ should be 4 times longer than that with HAS thickness of $20 \mu \mathrm{m}$. However, the experiment reveals more than 10 times faster bending speed in the latter sample. This may be caused by a reduction of the effect of Up-hill diffusion which disturbs hydrogen diffusion in a distorted host lattice. By thinning the sample thickness, the gradient of hydrogen concentration in the direction of sample thickness should be lowered and the distortion of the lattice may be reduced, and hence an obstruction for hydrogen diffusion is weakened.

\section{Conclusions}

Pd-Ni hydrogen storage alloys are suitable for an application to actuators using a volume expansion of a hydrogen storage alloy as driving force because of easy activation, plateau pressures of nearly $0.1 \mathrm{MPa}$ (atmospheric pressure) on hydrogen absorption-desorption cycles and a small hysteresis in the pressure-composition isotherm. The Cu-plated Pd-Ni alloy samples showed a bending motion on hydrogenation and also showed a recover of displacement in vacuo after the sample was held in air for 24 hours. This may be caused by an oxidation resistance of palladium alloy surface and a proper desorption plateau pressure, and it is a desirable characteristic in various application. By reducing the thickness of the alloy, bending speed increased largely and a large bending displacement can be obtained in a short time. However, a 
reduction in sample thickness will cause a lowering in its strength. It is necessary to modify the structure of bending module from single layered to multi-layered one for reinforcement of the module strength on application to a powerful actuator.

\section{Acknowledgements}

This research was partially supported by the Ministry of Education, Culture, Sports, Science and Technology, Grant-in-Aid for Priority areas, 19016019, 2007.

\section{References}

[1] Y. Ohsumi, Data book of Hydrogen Storage alloys, Yono-shobo, Tokyo, 1987, p.92.

[2] A. Kagawa, Reports of the Faculty of Engineering, Nagasaki University, 25(47) (1995) 223.

[3] A. Kagawa, E. Ono, T. Kusakabe, Y. Sakamoto, J. Alloys Comp., 172-174 (1995) 226.

[4] T. Honjo, H. Yabe, S. Tubuteishi, H. Uchida, Y Nishi, J. Japan Inst. Metals, 67(4) (2003) 145.

[5] T. Honjo, H. Uchida, Y. Matsumura, Y. Nishi, J. Japan Inst. Metals, 68(4) (2004) 58.

[6] T. B. Massalski, Binary Alloy Phase Diagrams, ASM, Metals Park, Ohio, 1987.

[7] B. Baranowski, F. A. Lewis, Ber. Bunsenges Phys. Chem., 93 (1984) 1225.

[8] B. Baranowski, J. Less-Common Met., 154 (1989) 329. 
Figure captions

Fig. 1 Bending-motion actuator using hydrogen storage alloy.

Fig. 2 Shape change measurement apparatus.

Fig. 3 Pressure-composition isotherm of Pd-11at\%Ni alloy.

Fig. 4 Bending behavior of Pd-11at\%Ni sample on hydrogen absorption.

Fig. 5 Relationship between displacement and time on cyclic hydrogenation for Pd11 at $\% \mathrm{Ni}$ alloy sample.

Fig. 6 Relationship between response time and cycle number for Pd-11at\%Ni alloy sample.

Fig. 7 Appearance of Pd-11at\%Ni alloy sample with HSA $20 \mu \mathrm{m}$ thick on hydrogenation.

Fig. 8 Variation in displacement of top end of the Pd-11at\%Ni alloy samples having different thickness of HAS with time. 


\section{Hydrogen storage alloy}

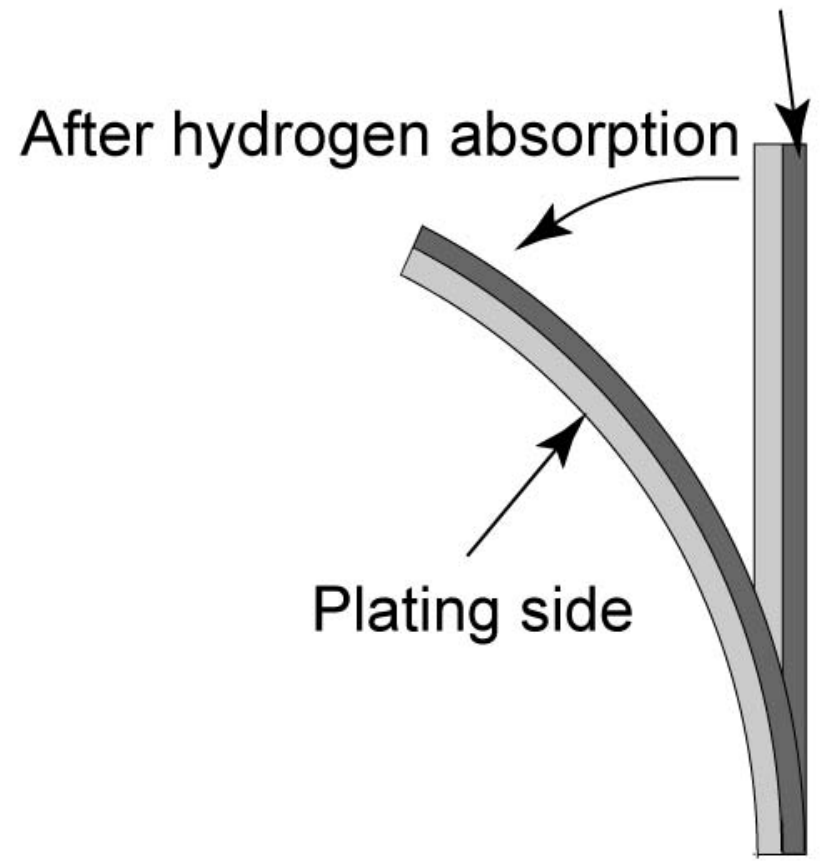

Fig. 1 Bending-motion actuator using hydrogen storage alloy. 




Fig. 2 Shape change measurement apparatus. 


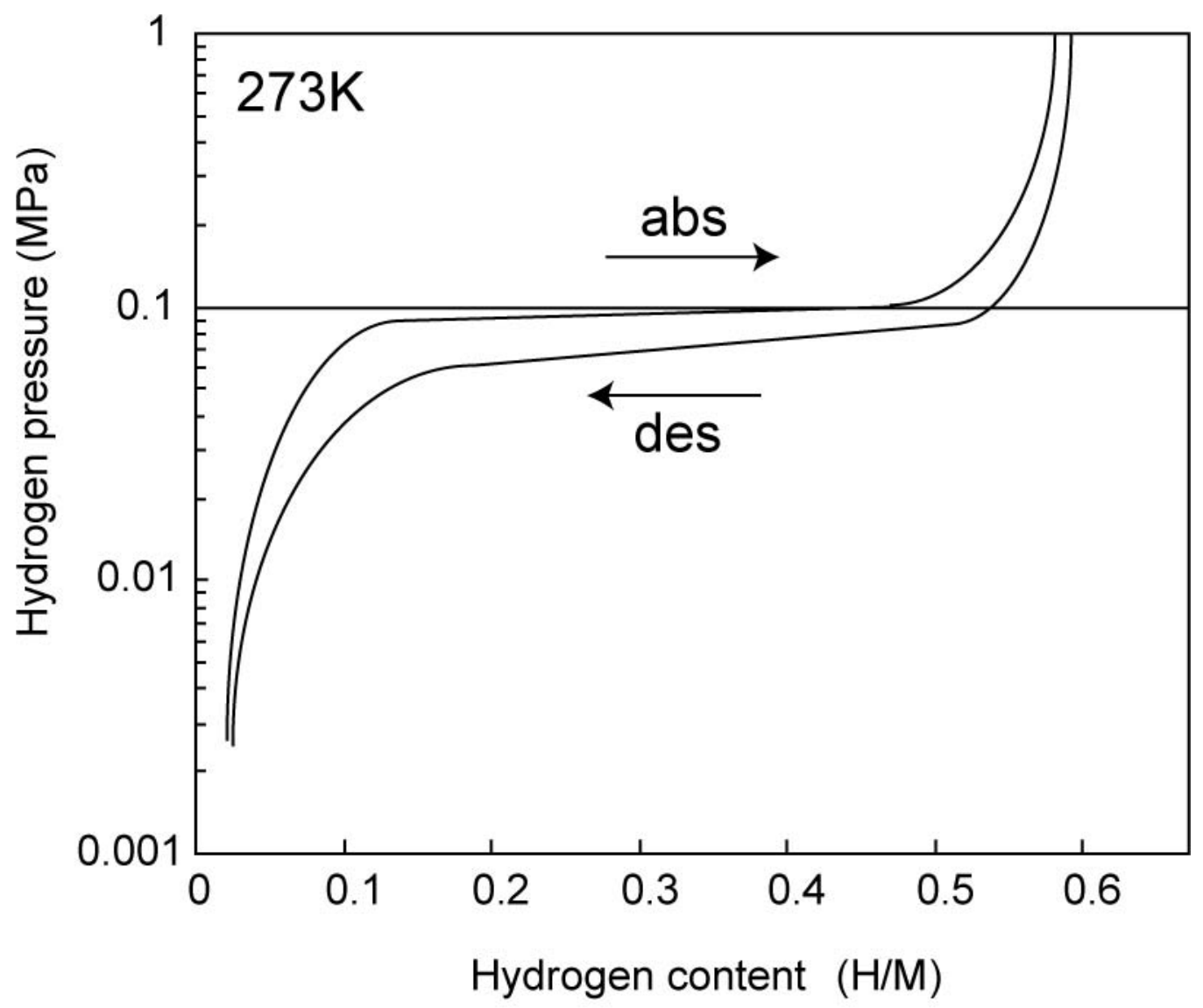

Fig. 3 Pressure-compsition isotherm of $\mathrm{Pd}-11$ at $\% \mathrm{Ni}$ alloy.
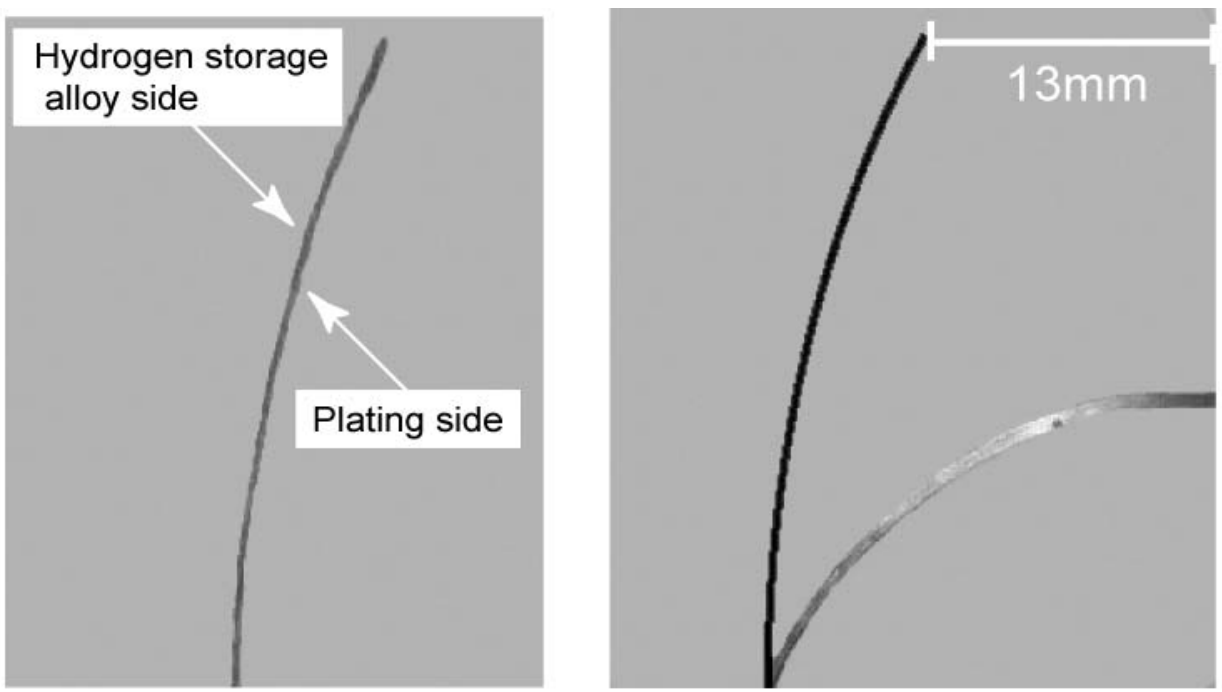

Fig. 4 Bending behavior of Pd-11at\%Ni sample on hydrogen absorption. 


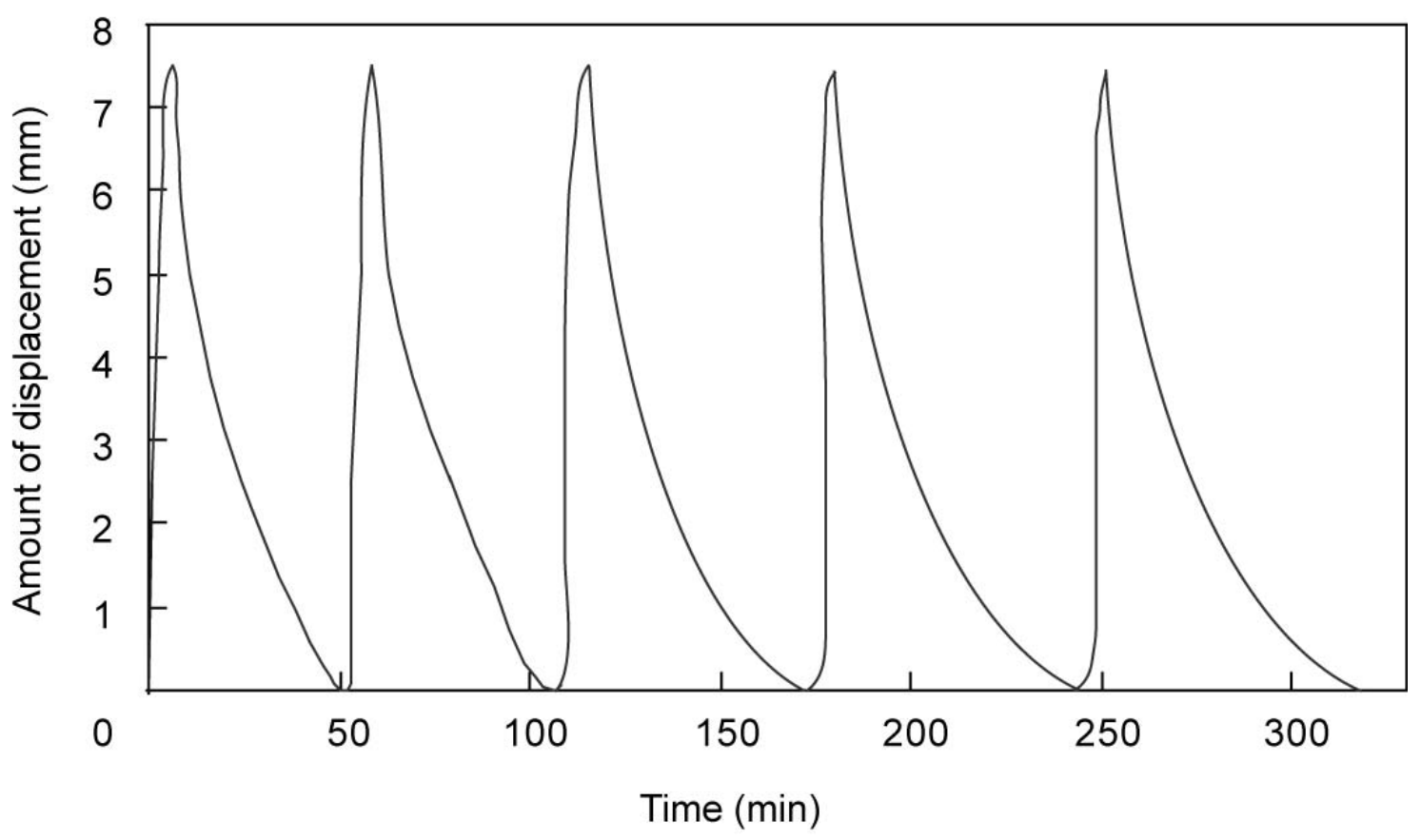

Fig. 5 Relationship between displacement and time on cyclic hydrogenation for Pd11 at $\%$ Ni alloy sample.

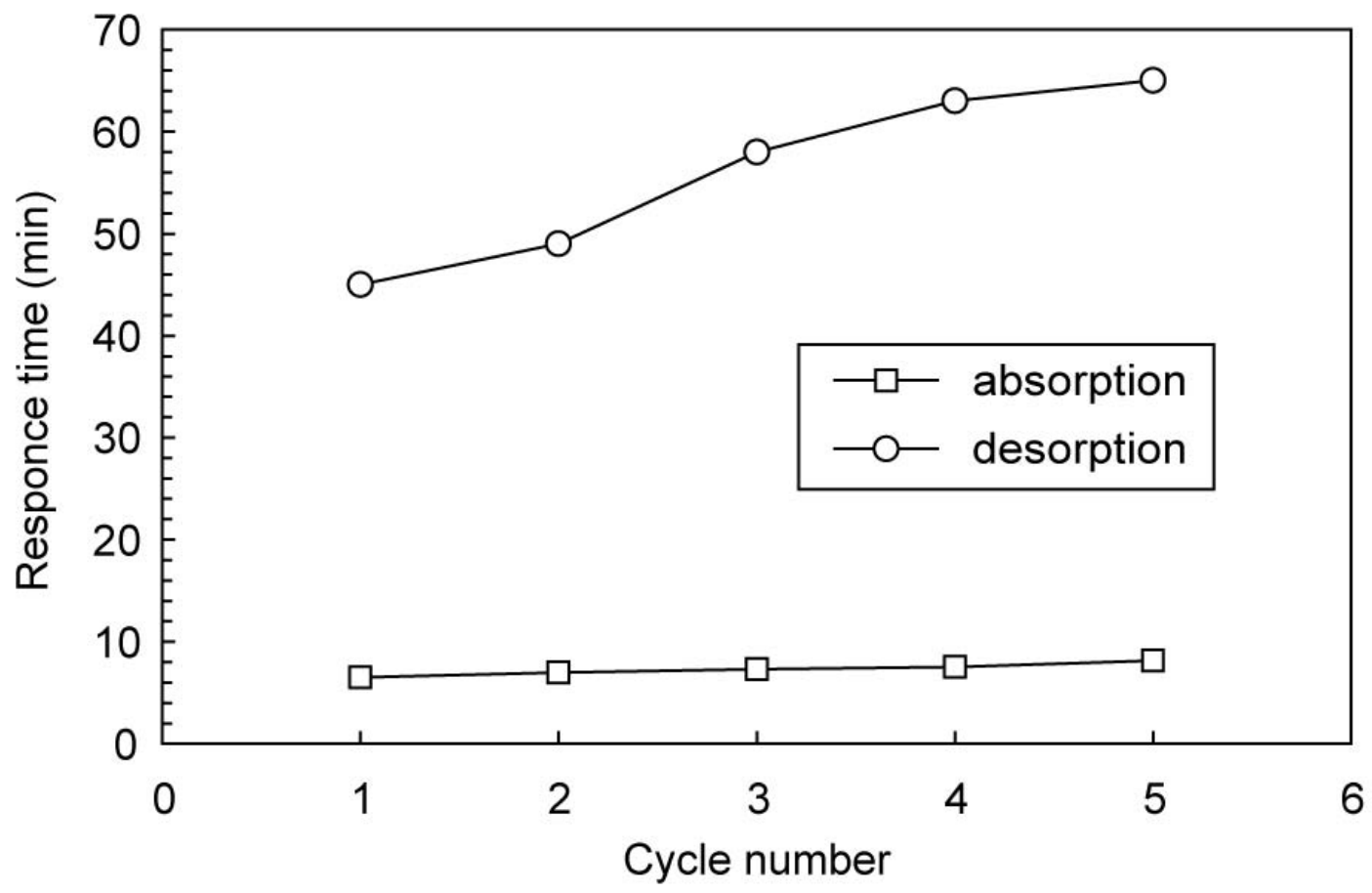

Fig. 6 Relationship between response time and cycle number for Pd-11at\%Ni alloy sample. 


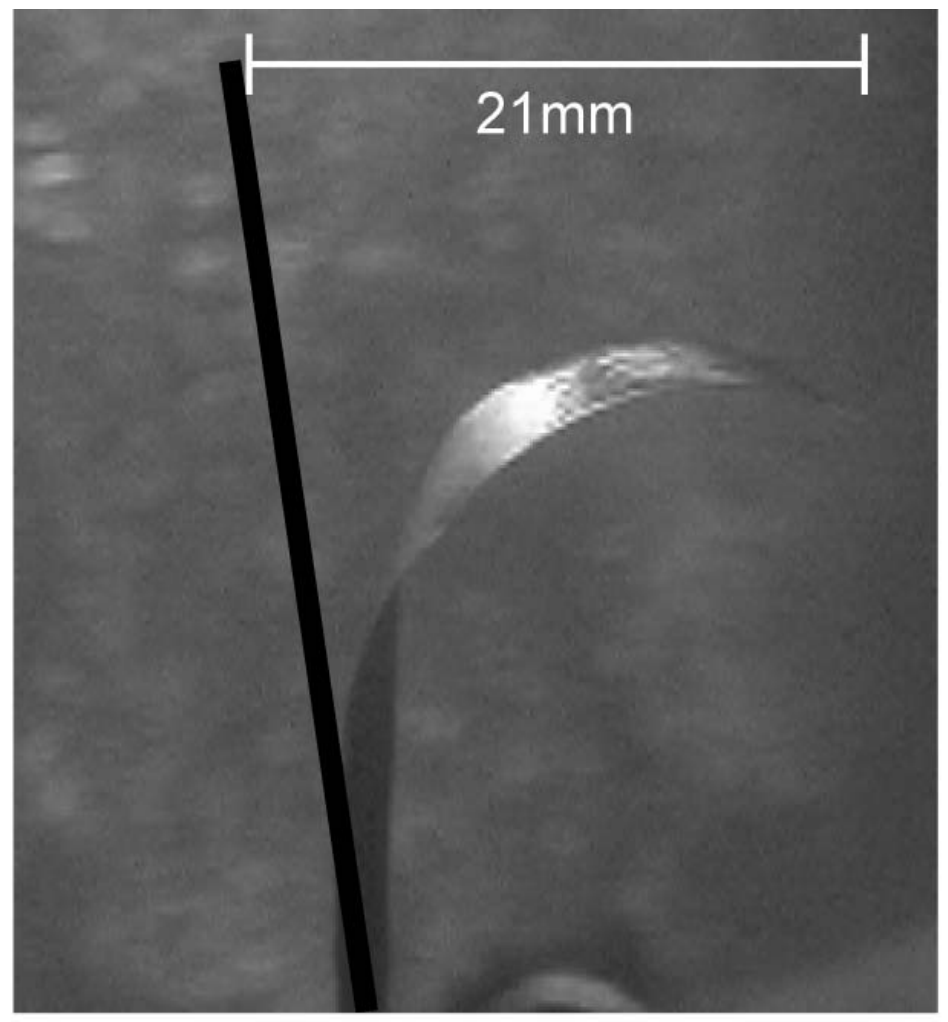

Fig. 7 Appearance of sample with HSA $20 \mu \mathrm{m}$ thick on hydrogenation. 


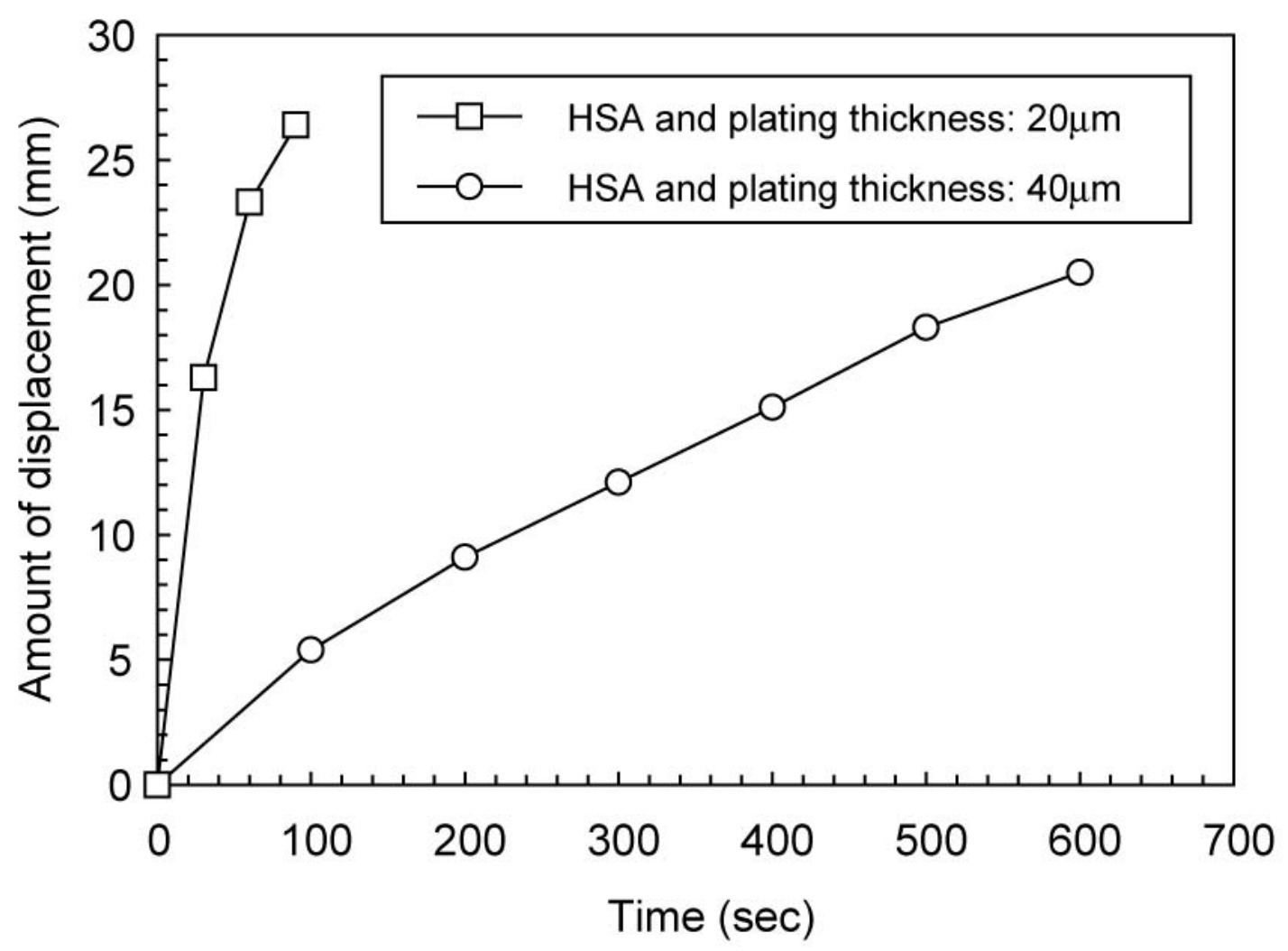

Fig. 8 Variation in displacement of top end of the Pd- 11 at $\% \mathrm{Ni}$ alloy samples having different thickness of HAS with time. 